\title{
The role of cardiovascular magnetic resonance in stratifying paravalvular leak severity after transcatheter aortic valve replacement: an observational outcome study
}

Gregory R Hartlage ${ }^{1,2}$, Vasilis C Babaliaros ${ }^{1}$, Vinod H Thourani ${ }^{3}$, Salim Hayek', Christina Chrysohoou', Nima Ghasemzadeh ${ }^{1}$, Arthur E Stillman ${ }^{2}$, Stephen D Clements ${ }^{1}$, John N Oshinski ${ }^{2,4}$ and Stamatios Lerakis ${ }^{1,2,4,5^{*}}$

\begin{abstract}
Background: Significant paravalvular leak (PVL) after transcatheter aortic valve replacement (TAVR) confers a worse prognosis. Symptoms related to significant PVL may be difficult to differentiate from those related to other causes of heart failure. Cardiovascular magnetic resonance (CMR) directly quantifies valvular regurgitation, but has not been extensively studied in symptomatic post-TAVR patients.

Methods: CMR was compared to qualitative (QE) and semi-quantitative echocardiography (SQE) for classifying PVL and prognostic value at one year post-imaging in 23 symptomatic post-TAVR patients. The primary outcome was a composite of all-cause death, heart failure hospitalization, and intractable symptoms necessitating repeat invasive therapy; the secondary outcome was a composite of all-cause death and heart failure hospitalization. The difference in event-free survival according to greater than mild PVL versus mild or less PVL by QE, SQE, and CMR were evaluated by Kaplan-Meier survival analysis.

Results: Compared to QE, CMR reclassified PVL severity in $48 \%$ of patients, with most patients (31\%) reclassified to at least one grade higher. Compared to SQE, CMR reclassified PVL severity in 57\% of patients, all being reclassified to at least one grade lower; SQE overestimated PVL severity (mean grade 2.5 versus 1.7, $p=0.001$ ). The primary and secondary outcomes occurred in $48 \%$ and $35 \%$ of patients, respectively. Greater than mild PVL by CMR was associated with reduced event-free survival for the primary outcome $(p<0.0001)$, however greater than mild PVL by QE and SQE were not $(p=0.83$ and $p=0.068)$. Greater than mild PVL by CMR was associated with reduced event-free survival for the secondary outcome, as well $(p=0.012)$.

Conclusion: In symptomatic post-TAVR patients, CMR commonly reclassifies PVL grade compared with QE and SQE. CMR provides superior prognostic value compared to QE and SQE, as patients with greater than mild PVL by CMR (RF > 20\%) had a higher incidence of adverse events.
\end{abstract}

Keywords: Cardiovascular magnetic resonance imaging, Paravalvular regurgitation, Prognosis, Transcatheter aortic valve implantation

\footnotetext{
*Correspondence: sleraki@emory.edu

'Department of Medicine, Division of Cardiology, Structural Heart and Valve

Center, Emory University School of Medicine, Atlanta, Georgia

${ }^{2}$ Department of Radiology and Imaging Science, Emory University School of Medicine, Atlanta, GA, Georgia

Full list of author information is available at the end of the article
} 


\section{Background}

Transcatheter aortic valve replacement (TAVR) has transformed the contemporary management of patients with severe aortic stenosis and high surgical risk [1,2]. Despite extensive pre-procedure evaluation, greater than mild paravalvular leak (PVL) occurs in over $10 \%$ of patients undergoing TAVR and is associated with worse short and long term outcomes $[3,4]$. Echocardiography, the standard non-invasive method of imaging PVL, often has limited utility due to multiple eccentric regurgitant jets [5]. Acoustic shadowing from the valve stent and native aortic valve calcification may further complicate the estimation of PVL. The Valve Academic Research Consortium II (VARC II) has proposed a semi-quantitative method of PVL classification by the extent of circumferential involvement relative to the prosthetic valve stent on two-dimensional echocardiography [6], which is yet to be validated.

Accurate identification of post-TAVR PVL by cardiac imaging is essential, as symptoms from PVL may be difficult to clinically differentiate from symptoms related to other common causes of heart failure, such as systolic and diastolic dysfunction. Cardiovascular magnetic resonance (CMR) flow assessment provides accurate and reproducible quantification of valvular regurgitation $[7,8]$. The direct measurement of forward and reverse flow volumes by CMR facilitates the calculation of the aortic regurgitant fraction (RF) for severity classification. Small studies have demonstrated the feasibility of CMR for evaluation of post-TAVR PVL [9-11], however, these studies did not address diagnostic and prognostic value in symptomatic patients. The purpose of our study was to evaluate CMR in post-TAVR patients in whom there was concern for clinically significant PVL. In addition to PVL classification, we assessed the ability of CMR findings to predict outcomes at one year post-imaging.

\section{Methods \\ Study population}

The study was approved and performed in accordance with the regulations of the university's institutional review board (Emory University Hospital, Atlanta, Georgia). We retrospectively reviewed all patients at our center from 2009 to 2013 that underwent CMR post-TAVR. Patients had been implanted with either a balloonexpandable or self-expanding transcatheter aortic prosthesis. We included all patients undergoing CMR for evaluation of heart failure symptoms persisting or recurring after TAVR, which were potentially related to PVL seen on transthoracic echocardiography (TTE). A detailed medical history, laboratory values and TTE findings were collected to establish baseline demographics prior to TAVR and prior to CMR.

\section{Echocardiography}

TTE was performed on a Phillips iE33 (Leiden, The Netherlands) or General Electric Vivid 7 (Milwaukee, Wisconsin) as the initial test for the evaluation of PVL. PVL was classified qualitatively by visual assessment at the point of care, as well as semi-quantitatively by VARC II criteria post-hoc. Qualitative echocardiography $(\mathrm{QE})$ included visual estimation of the width and area of the color Doppler jet in the left ventricular outflow tract in the parasternal short and long-axis views and was graded mild (jet width $<25 \%$ of left ventricular outflow tract width), moderate (jet width 25 to $65 \%$ of left ventricular outflow tract width), and severe (jet width $>65 \%$ of left ventricular outflow tract width). For semi-quantitative echocardiography (SQE), the VARC II PVL classification scheme was employed using the circumferential extent of the regurgitant jet(s) in the parasternal short-axis (the sum of the PVL jet circumferences divided by the valve circumference; graded as mild $<10 \%$, moderate 10 to $30 \%$, severe $>30 \%$; see Figure 1). Suprasternal notch and subcostal views of the descending aorta and parasternal short and long-axis view of the right ventricular outflow tract and main pulmonary artery were consistently attempted.

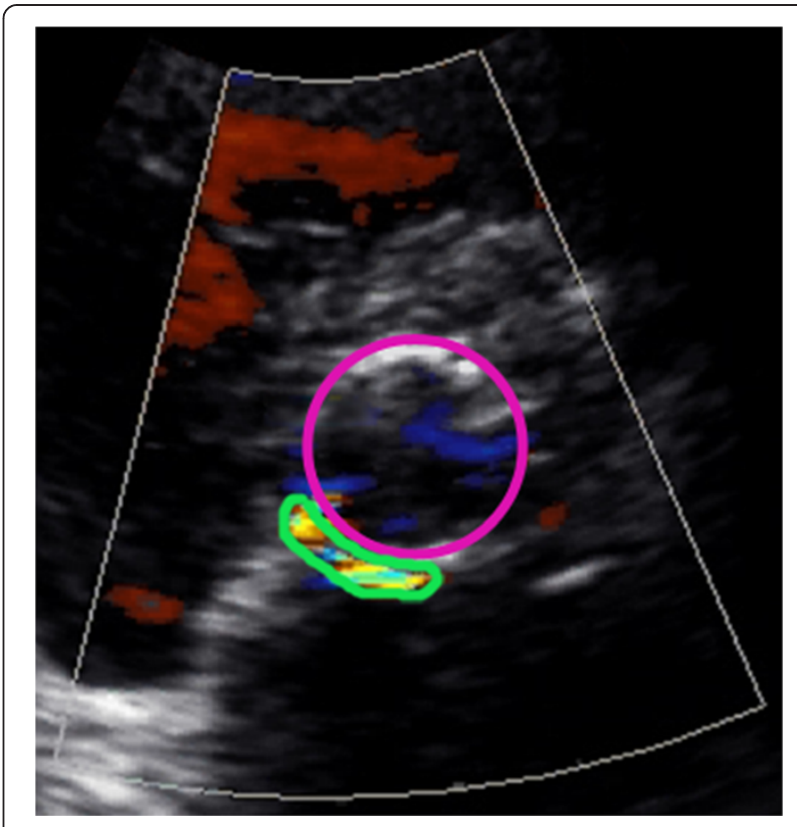

Figure 1 Semi-quantitative echocardiographic (SQE) measurement of paravalvular leak (PVL) using circumferential extent of PVL in the parasternal short-axis by the Valve Academic Research Consortium II method. The green circle represents the circumference of the paravalvular leak. The purple circle represents the circumference of the transcatheter valve stent frame. The circumferential extent is the sum of the paravalvular leak jet circumference(s) divided by the valve circumference (mild $<10 \%$, moderate $10-30 \%$, severe $>30 \%$ ). 


\section{Cardiovascular magnetic resonance acquisition}

CMR was performed on a Siemens Avanto 1.5 T scanner (Erlangen, Germany) using a 5 element phased array coil. Velocity-encoded phase-contrast magnetic resonance (PCMR) imaging was utilized in the ascending aorta for flow quantification [12]. The scan plane was placed perpendicular to the long-axis of the proximal ascending aorta 2-3 $\mathrm{mm}$ above the valve stent frame for through-plane flow measurement in patients with a balloon-expandable prosthesis (see Figure 2A) and at a similar position through the non-ferromagnetic stent frame in patients with a selfexpanding prosthesis. Free-breathing acquisitions were used preferentially, including patients with irregular heart rhythms, with breath-hold acquisitions reserved for patients with respiratory image artifacts due to irregular breathing. Electrocardiographic triggering was used to obtain 20 frames per cardiac cycle for patients with a regular heart rhythm, while free-breathing real time acquisitions with multiple signal averaging was used for patients with an irregular heart rhythm; the slice thickness was $6 \mathrm{~mm}$. The VENC was initially set at 200-250 cm/s with routine VENC optimization.

\section{Cardiovascular magnetic resonance analysis}

For the assessment of aortic RF, the cross sectional area of the aorta was traced throughout the cardiac cycle on each separate magnitude image to define regions of interest (see Figure 2B). The forward and reverse flow volumes were calculated within the corresponding region of interest on the phase image (see Figure 2C) by offline analysis using Siemens Argus software (Erlangen, Germany). Background phase offset correction was done by using a region of static tissue near the ascending aorta. Aortic RF was calculated as: reverse aortic flow volume divided by forward aortic flow volume; see Figure 2D. CMR PVL severity was classified by regurgitant fraction (RF; mild $\leq 20 \%$, moderate 21 to $39 \%$, severe $\geq 40 \%$ ).

\section{PVL classification comparison}

The classification of PVL was compared using three modalities in all patients: QE, SQE, and CMR (used as the gold standard for this study). By convention, PVL was classified as 1 (mild), 2 (moderate) and 3 (severe) for analysis. Inter-method agreement of classification was analyzed within each patient and for the whole group.
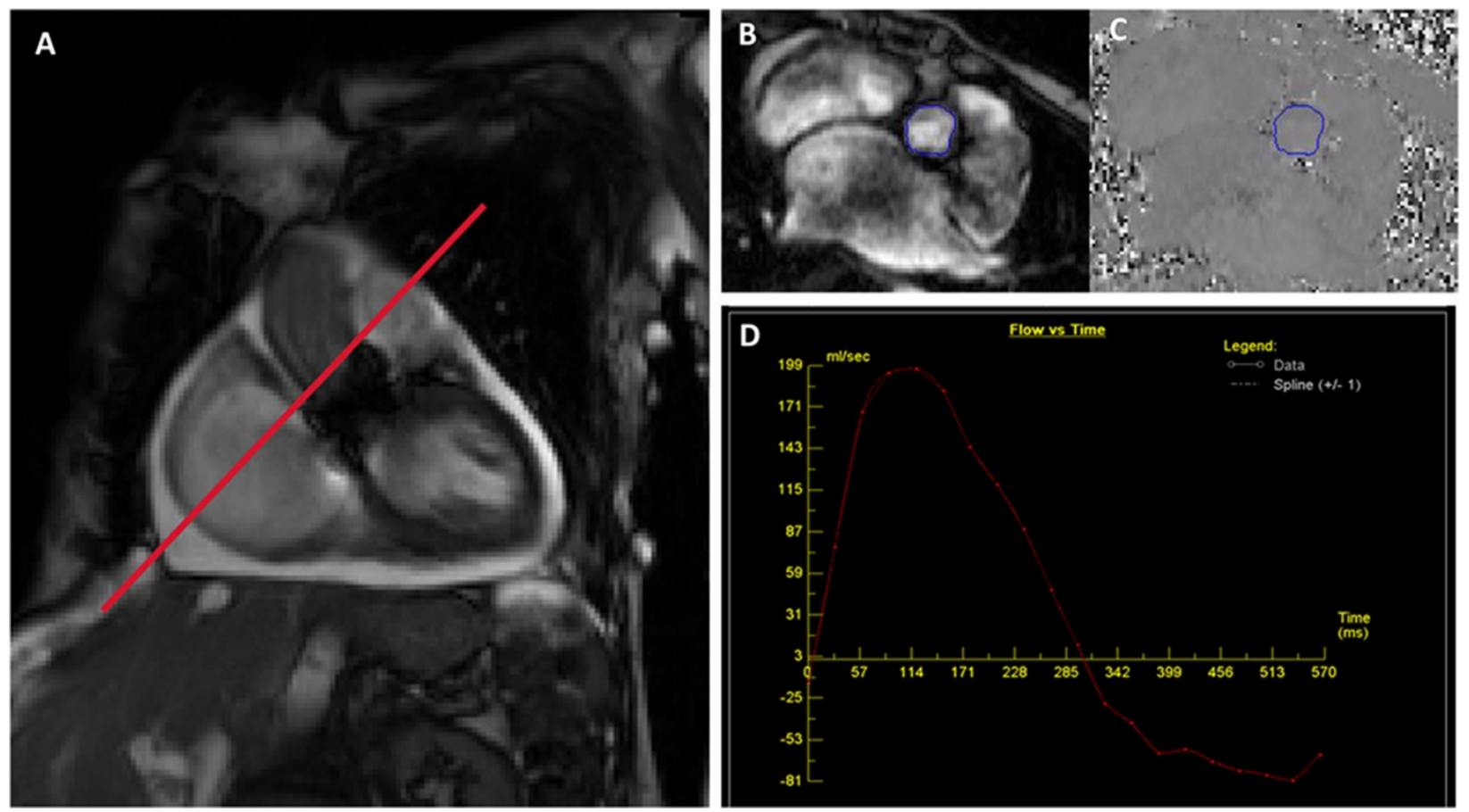

Figure 2 Measurement of paravalvular leak (PVL) regurgitant fraction (RF) by cardiovascular magnetic resonance (CMR). The positioning of the scan plane is demonstrated for the balloon-expandable prosthesis in the aortic root 2-3 $\mathrm{mm}$ above the valve stent frame (A). The regions of interest are traced on the magnitude images (anatomical scan; $\mathbf{B}$ ) and the phase images (flow scan; $\mathbf{C}$ ). The regions of interest include the entire intra-luminal, cross sectional area of flow just above the transcatheter valve. The flow through the region of interest is calculated throughout the cardiac cycle (D), with the area under the curve (above baseline) representing forward flow volume and the area above the curve (below baseline) representing reverse flow volume. The aortic regurgitant fraction was calculated by dividing the reverse flow volume by the forward flow volume (mild $\leq 20 \%$, moderate $21-39 \%$, severe $\geq 40 \%$ ). 
We documented reclassification of PVL grade by CMR relative to QE and SQE. PVL severity was considered downgraded if the CMR classification was $\geq 1$ grade lower and upgraded if $\geq 1$ grade higher as compared to each TTE method.

\section{Outcomes}

Study outcomes were determined by retrospective chart review. All patients in this analysis were enrolled in prospective TAVR trials or registries with long-term followup and data collection at our clinic. Follow-up included detailed interval histories including any hospital admissions or procedures taking place at Emory University Hospital or outside healthcare facilities. Any patient deaths not taking place at Emory University Hospital were reported to clinic study personnel and documented in the chart, as well. The primary composite outcome was the combination of all-cause death, heart failure hospitalization, and intractable heart failure symptoms necessitating repeat invasive therapy at one-year followup. The secondary composite outcome included the combined endpoints of all-cause death and heart failure hospitalization. Intractable heart failure included New York Heart Association (NYHA) class III and IV symptoms despite aggressive oral diuretic and/or vasodilator therapy. Repeat invasive therapy for significant PVL included repeat balloon valve dilation, transcatheter PVL plug placement, transcatheter valve-in-valve placement, or surgical valve replacement. Heart failure hospitalization included any inpatient admission for congestive symptoms including worsening dyspnea and peripheral edema during which the patient was treated with intravenous diuretic and/or vasodilator therapy.

\section{Statistics}

Statistical analysis was performed with SPSS version 20 (Chicago, Illinois) and SAS version 9.3 (Cary, North Carolina). Descriptive results were expressed as numbers and percentages, while continuous variables were expressed as mean \pm standard deviation (unless otherwise stated). Continuous variables were analyzed by the independent-samples $\mathrm{t}$-test. Categorical variables were analyzed by the Fisher's exact test. PVL classification by each method was compared within patients by Spearman rho bivariate correlation and the groups were compared by the paired-samples Wilcoxon rank-sum test. Baseline risk was compared across mild, moderate, and severe PVL by CMR with the Kruskal-Wallis test to evaluate for significant differences between the groups. The difference in event-free survival according to greater than mild PVL versus mild or less PVL by QE, SQE, and CMR were evaluated by Kaplan-Meier survival analysis with Mantel-Cox log-rank. Inter-observer variability was assessed with intraclass correlation for continuous variables and Cohen's kappa coefficient for dichotomous classification (severity greater than mild versus mild or less). Statistical significance was determined by $\mathrm{p}<0.05$ on two-tailed analysis.

\section{Results \\ Patients}

CMR was performed in 23 post-TAVR patients with NYHA class III-IV symptoms (median 6 days [interquartile range $2-100$ days] post-TAVR and median 2 days [interquartile range 0-7 days] after index echocardiogram). The baseline Society for Thoracic Surgeons Predicted Risk of Mortality score was not significantly different among patients with mild, moderate, and severe PVL by CMR $(p=0.26)$. Baseline patient characteristics at the time of TAVR are presented in Table 1 and interval clinical and imaging characteristics at the time of CMR in Table 2. Patients with significant PVL with $\mathrm{RF}>20 \%$ on CMR were more likely to have atrial fibrillation at baseline. At the time of CMR, patients with a $\mathrm{RF}>20 \%$ had higher serum creatinine, higher serum brain-natriuretic peptide levels, larger PVL circumferential extent, and higher prevalence of other valve dysfunction of at least moderate degree (mitral and tricuspid regurgitation). Otherwise, there were no significant baseline differences at the time of TAVR or at the time of CMR in those with $R F>20 \%$ versus $R F \leq 20 \%$.

\section{Paravalvular leak classification by echocardiography and CMR}

All patients had adequate parasternal short-axis images on echocardiogram for semi-quantitative analysis. Suprasternal notch and subcostal views of the descending aorta were not consistently of diagnostic quality to assess diastolic flow reversal. Parasternal short and long-axis views of the right ventricular outflow tract and pulmonary artery were also not consistently of diagnostic quality to perform quantitative Doppler analysis. PVL classification by QE, SQE, and CMR is shown in Figure 3. Greater than mild PVL was present in $52 \%, 83 \%$ and $52 \%$ of patients by QE, $\mathrm{SQE}$, and CMR, respectively. There was a poor correlation between QE and CMR (Spearman $r=0.26, p=0.24$ ) and a moderate correlation between SQE and CMR (Spearman $r=0.59 ; \mathrm{p}=0.003)$. Mean PVL severity was not significantly different by QE and CMR, however, SQE overestimated severity compared to CMR (see Figure 4). PVL was reclassified by CMR in a substantial number of patients compared with QE and SQE (see Table 3).

\section{Primary composite outcome}

At one year (mean $11.5 \pm 1.4$ months), $48 \%$ of the patients met the primary composite outcome of all-cause death, heart failure hospitalization, and intractable heart failure symptoms necessitating repeat invasive therapy 
Table 1 Baseline patient characteristics at TAVR*

\begin{tabular}{|c|c|c|c|c|}
\hline & All $(n=23)$ & RF $<20 \%(n=11)$ & $R F>20 \%(n=12)$ & $P$ value \\
\hline Male & $16(70)$ & $6(55)$ & $10(83)$ & 0.19 \\
\hline Age $($ mean $\pm S D)$ & $83 \pm 6$ & $83 \pm 7$ & $82 \pm 5$ & 0.86 \\
\hline NYHA class (mean \pm SD) & $3.2 \pm 0.7$ & $3.1 \pm 0.7$ & $3.3 \pm 0.8$ & 0.61 \\
\hline STS-PROM (\%; mean \pm SD) & $6.9 \pm 2.4$ & $6.4 \pm 2.0$ & $7.4 \pm 2.7$ & 0.33 \\
\hline Body mass index $\left(\mathrm{kg} / \mathrm{m}^{2}\right)$ & $25 \pm 4$ & $25 \pm 4$ & $24 \pm 4$ & 0.64 \\
\hline \multicolumn{5}{|l|}{ Comorbidities } \\
\hline Coronary artery disease & $13(57)$ & $7(64)$ & $6(50)$ & 0.68 \\
\hline CABG & $7(30)$ & $2(18)$ & $5(42)$ & \\
\hline $\mathrm{PCl}$ & $8(35)$ & $4(36)$ & $4(33)$ & \\
\hline Myocardial infarction & $7(30)$ & $3(27)$ & $4(33)$ & \\
\hline Atrial fibrillation & $10(44)$ & $2(18)$ & $8(67)$ & 0.04 \\
\hline Diabetes mellitus & $10(44)$ & $5(46)$ & $5(42)$ & 1.00 \\
\hline Hypertension & $21(91)$ & $10(91)$ & $11(92)$ & 1.00 \\
\hline Serum creatinine $>1.5 \mathrm{mg} / \mathrm{dl}$ & $8(35)$ & $2(18)$ & $6(50)$ & 0.19 \\
\hline COPD & $8(35)$ & $3(27)$ & $5(42)$ & 0.67 \\
\hline \multicolumn{5}{|l|}{ Laboratory } \\
\hline Creatinine (mg/dl; median [IQR]) & $1.11(0.90,1.61)$ & $1.07(1.00,1.30)$ & $1.45(0.86,1.89)$ & 0.18 \\
\hline BNP (pg/ml; median [IQR]) & $545(226,1612)$ & $297(197,843)$ & $1385(360,1806)$ & 0.17 \\
\hline \multicolumn{5}{|l|}{ Echocardiography } \\
\hline Ejection fraction (\%; mean \pm SD) & $47 \pm 14$ & $45 \pm 13$ & $49 \pm 15$ & 0.46 \\
\hline Aortic insufficiency* $($ mean \pm SD) & $1.1 \pm 0.7$ & $1.0 \pm 0.7$ & $1.1 \pm 0.7$ & 0.90 \\
\hline RVSP $(\mathrm{mmHg} ;$ mean $\pm \mathrm{SD})$ & $48 \pm 17$ & $43 \pm 12$ & $53 \pm 19$ & 0.17 \\
\hline RVSP $>55 \mathrm{mmHg}$ & $7(30)$ & $2(18)$ & $5(42)$ & 0.37 \\
\hline Transfemoral approach & $17(74)$ & $6(55)$ & $11(92)$ & 0.07 \\
\hline
\end{tabular}

*All results are presented as $\mathrm{n}(\%)$ unless otherwise noted.

$\mathrm{BNP}=$ brain natriuretic peptide, $\mathrm{CABG}=$ coronary artery bypass grafting, $\mathrm{COPD}=$ chronic obstructive pulmonary disease, IQR $=$ interquartile range, $\mathrm{NYHA}=\mathrm{New}$ York Heart Association, $\mathrm{PCl}=$ percutaneous coronary intervention, RVSP = right ventricular systolic pressure, STS-PROM = Society of Thoracic Surgeons predicted risk of mortality, TAVR = transcatheter aortic valve replacement.

(see Table 4). The average time to first event was 51 days ( 9 days for repeat invasive therapy, 97 days for heart failure admission, and 118 days for all-cause death). Patients who experienced the primary outcome had significantly higher serum creatinine levels at the time of CMR $(1.9 \pm$ $0.80 \mathrm{mg} / \mathrm{dl}$ versus $1.17 \pm 0.32 \mathrm{mg} / \mathrm{dl}, \mathrm{p}=0.008$ ) and higher EF by CMR ( $53 \pm 10 \%$ versus $44 \pm 12 \%, \mathrm{p}=0.048)$. The primary outcome occurred in $9 \%, 71 \%$, and $100 \%$ of patients with mild, moderate, and severe PVL by CMR, respectively. Patients with greater than mild PVL by CMR were more likely to experience the primary outcome compared to those with mild or less PVL ( $\mathrm{p}=0.001$ ), while patients with greater than mild PVL by QE or SQE were not $(\mathrm{p}=1.0$ and $\mathrm{p}=0.093$, respectively). Otherwise, there were no significant baseline differences in patients with and without a primary outcome event. Severity of PVL by QE, SQE, and CMR in those with and without a primary outcome event is shown in Figure 5. Kaplan-Meier survival analysis stratified by greater than mild PVL stratified by $\mathrm{QE}, \mathrm{SQE}$, and CMR is shown in Figure 6.

\section{Secondary composite outcome}

At one year, $35 \%$ of patients met the secondary composite outcome of all-cause death and heart failure hospitalization. The average time to heart failure admission was 109 days and average time to death was 150 days. The secondary outcome occurred in $9 \%, 57 \%$, and $60 \%$ of patients with mild, moderate, and severe PVL by CMR, respectively. Patients with greater than mild PVL by CMR were significantly more likely to experience a secondary composite outcome event compared to those with mild or less PVL $(p=0.027)$, while patients with greater than mild PVL by QE or SQE were not $(\mathrm{p}=0.19$ and $\mathrm{p}=0.26$, respectively). Otherwise, there were no significant differences in patients with and without a secondary outcome event. Kaplan-Meier survival analysis stratified by greater than mild PVL by QE, SQE, and CMR is shown in Figure 7.

\section{Reproducibility analysis}

Inter-observer variability for $\mathrm{QE}, \mathrm{SQE}$ and CMR was assessed by 2 independent readers (G.H. and S.L.) in 10 
Table 2 Patient characteristics at time of CMR*

\begin{tabular}{|c|c|c|c|c|}
\hline & All $(n=23)$ & $\mathrm{RF}<20 \%(n=11)$ & $\mathrm{RF}>20 \%(n=12)$ & $\mathrm{P}$ value \\
\hline Age (mean $\pm S D)$ & $83 \pm 6$ & $83 \pm 7$ & $83 \pm 5$ & 0.82 \\
\hline NYHA class (mean \pm SD) & $3.5 \pm 0.8$ & $3.4 \pm 0.9$ & $3.7 \pm 0.7$ & 0.37 \\
\hline$\triangle$ NYHA class since TAVR (median[IQR]) & $1(0,1)$ & $1(-1,1)$ & $0(0,1)$ & 0.77 \\
\hline STS-PROM (\%; mean \pm SD) & $7.8 \pm 4.6$ & $6.3 \pm 2.3$ & $9.0 \pm 5.7$ & 0.16 \\
\hline$\triangle S T S-P R O M$ since TAVR (\%; median[IQR]) & $0(-0.5,1.7)$ & $0(-1.1,0)$ & $0.8(-0.4,3.0)$ & 0.15 \\
\hline \multicolumn{5}{|l|}{ Laboratory } \\
\hline Creatinine (mg/dl; median[IQR]) & $1.29(1.00,1.84)$ & $1.11(1.00,1.37)$ & $1.61(1.06,2.63)$ & 0.011 \\
\hline$\Delta$ Creatinine since TAVR (mg/dl; median[IQR]) & $0.1(-0.06,0.39)$ & $0.03(-0.08,0.19)$ & $0.36(0.10,0.55)$ & 0.13 \\
\hline BNP (pg/ml; median[IQR])) & $496(252,1335)$ & $432(235,546)$ & $1038(339,1948)$ & 0.05 \\
\hline$\triangle B N P$ since TAVR (pg/ml; median[IQR]) & $0(-419,199)$ & $0(-411,85)$ & $68(-441,240)$ & 0.73 \\
\hline \multicolumn{5}{|l|}{ Echocardiography } \\
\hline Circumferential extent (\%; mean \pm SD) & $34.2 \pm 18.4$ & $23.3 \pm 18.2$ & $44.3 \pm 11.9$ & 0.003 \\
\hline \multicolumn{5}{|l|}{ CMR } \\
\hline Ejection fraction (\%; mean \pm SD) & $48 \pm 12$ & $47 \pm 10$ & $49 \pm 14$ & 0.78 \\
\hline Other valve disease greater than mild $\dagger$ & $14(61)$ & $4(36)$ & $10(83)$ & 0.04 \\
\hline
\end{tabular}

*All results are presented as $\mathrm{n}(\%)$ unless otherwise noted.

tIncludes mitral and tricuspid regurgitation.

$\mathrm{BNP}=$ brain natriuretic peptide, $\mathrm{CMR}=$ cardiovascular magnetic resonance, $\mathrm{IQR}=$ interquartile range, NYHA= New York Heart Association, $\mathrm{PVL}=$ paravalvular leak, RF = regurgitant fraction, STS-PROM = Society of Thoracic Surgeons predicted risk of mortality, TAVR= transcatheter aortic valve replacement, $\mathrm{TTE}=$ transthoracic echocardiography.

randomly selected patients for each method. For QE, the intraclass correlation coefficient for PVL severity by visual grading was $0.61(\mathrm{p}=0.022)$, with excellent agreement between readers for PVL classification (Cohen's kappa coefficient 1.0, $\mathrm{p}=0.002$ ). For $\mathrm{SQE}$, the intraclass correlation coefficient for PVL circumferential extent by the VARC II method was $0.58(\mathrm{p}=0.030)$, with good agreement between readers for PVL classification (Cohen's kappa coefficient $0.60, \mathrm{p}=0.016$ ). For CMR, the intraclass correlation coefficient for PVL regurgitant fraction was $0.93(\mathrm{p}<0.0001)$ with excellent agreement between readers for PVL classification (Cohen's kappa coefficient 0.82, p < 0.0001).

\section{Discussion}

We present an early experience demonstrating the added value of CMR for diagnostic classification and risk stratification in symptomatic patients with post-TAVR PVL. PVL classification by PCMR flow quantification was reproducible and provided prognostic value superior to both qualitative and semi-quantitative echocardiography, suggesting that more widespread use of CMR derived PVL quantification may be warranted in symptomatic postTAVR patients. Flow quantification by PCMR is a wellestablished method for evaluating proximal aortic forward and reverse volumes $[7,8]$. Aortic regurgitation quantification is more reproducible by CMR compared to echocardiography for native valve disease $[13,14]$, however, it is noteworthy that quantitative RF values of aortic regurgitation severity obtained by PCMR are systematically lower than those obtained by echocardiography $[15,16]$. CMR derived quantitative findings have also shown prognostic value in native aortic regurgitation patients [17]. We present the first study evaluating the prognostic value of CMR derived PVL quantification in post-TAVR patients, in addition to diagnostic reclassification of PVL by CMR relative to various echocardiographic techniques.

CMR has demonstrated feasibility for the quantification of post-TAVR PVL [9-11]. In contrast to native aortic valve regurgitation, post-TAVR PVL is underestimated by QE compared to CMR $[10,18,19]$. Sherif et al. initially showed that QE underestimated the degree of PVL by $\geq 1$ grade in 7 of 16 (44\%) patients compared to CMR [10]. Recently, a multiparametric TTE approach was found to underestimate the degree of PVL by $\geq 1$ grade relative to CMR to a varying extent: 15 of 65 (23\%) patients in Orwat et al. [18] and 26 of 42 (62\%) patients in Ribiero et al. [19]. These findings are consistent with our study, in which QE identified no cases of severe PVL and PVL severity was upgraded by CMR by $\geq 1$ grade in nearly one-third of patients. A notable difference between our study and those above is that they utilized routine CMR screening in postTAVR patients regardless of symptoms, while our study evaluated CMR as a strategy to clarify post-TAVR symptoms. Importantly, we demonstrated limited prognostic power of QE while CMR was a strong predictor of adverse events, which highlights the clinical importance of the discrepancies between PVL grading with these modalities.

It is generally accepted that greater than mild PVL by echocardiography is associated with worse patient outcomes post-TAVR [3,4]. However, both transthoracic 


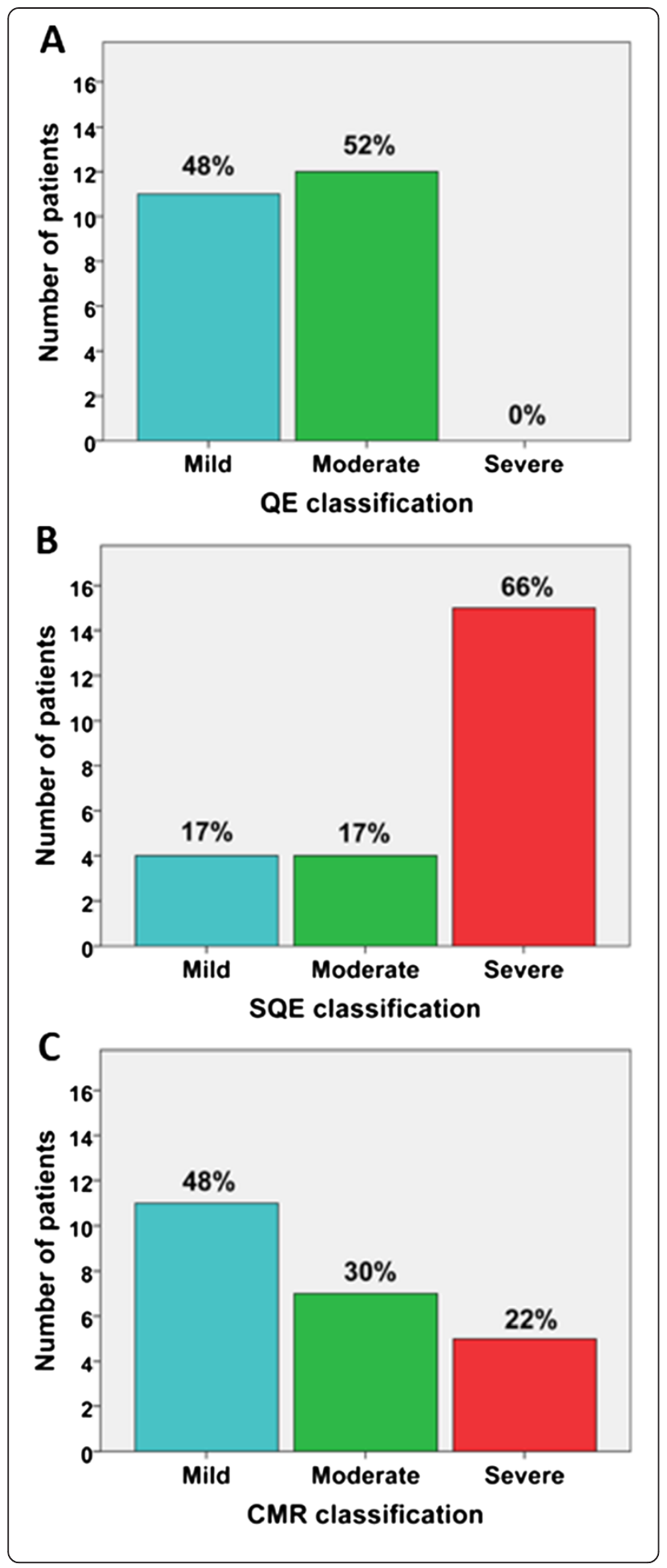

Figure 3 Comparison of paravalvular leak (PVL) classification by qualitative echocardiography (QE), semi-quantitative echocardiography (SQE), and cardiovascular magnetic resonance (CMR). QE classification included the estimated width of the color Doppler jet in the left ventricular outflow tract: mild (jet width $<25 \%$ of left ventricular outflow tract width), moderate (jet width 25 to $65 \%$ of left ventricular outflow tract width), and severe (jet width $>65 \%$ of left ventricular outflow tract width). SQE included the circumferential extent as the sum of the paravalvular leak jet circumference(s) divided by the valve circumference: mild $(<10 \%)$, moderate $(10-30 \%)$, and severe $(>30 \%)$. CMR classification included the aortic regurgitant fraction as calculated by dividing the reverse flow volume by the forward flow volume: mild ( $\leq 20 \%)$, moderate (21-39\%), and severe $(\geq 40 \%)$.

and transesophageal echocardiography may have significant limitations for the characterization and quantification of post-TAVR PVL. These limitations are related to two-dimensional anatomic views, angle dependence of Doppler assessment, multiple eccentric jets, and signal attenuation due to native calcification and implanted prosthetic material. Any combination of these factors can result in PVL underestimation. Such underestimation of post-TAVR PVL by QE may have led to previous findings implying that even mild post-TAVR PVL is associated with worse outcomes [1]. In our study, PVL severity by QE did not predict patient outcomes, further highlighting the limitations of QE in risk stratifying post-TAVR PVL. The assessment of PVL by PCMR overcomes the limitations of anatomic views and visual assessment to provide a truly quantitative evaluation of retrograde diastolic flow in the proximal aorta. Importantly, PCMR is technically feasible for PVL assessment in the balloon-expandable and self-

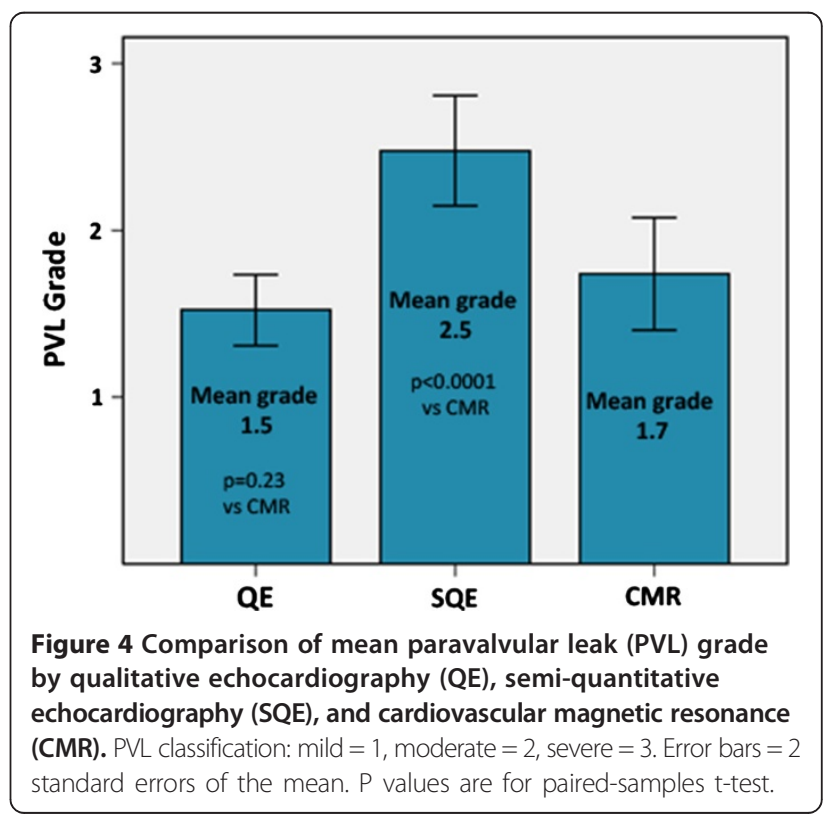


Table 3 Number of patients in which the PVL grade was reclassified after performing CMR

\begin{tabular}{ll}
\hline & All $\mathbf{n}=\mathbf{2 3}(\%)$ \\
\hline Qualitative echocardiography reclassification & $11(48)$ \\
Upgrade & $7(31)$ \\
Downgrade & $4(17)$ \\
Semi-quantitative echocardiography reclassification & $13(57)$ \\
Upgrade & $0(0)$ \\
Downgrade & $13(57)$ \\
\hline
\end{tabular}

$\mathrm{CMR}=$ cardiovascular magnetic resonance, $\mathrm{PVL}$ paravalvular leak, $\mathrm{RF}=$ regurgitant fraction, $\Pi \mathrm{TE}=$ transthoracic echocardiography.

expanding valves currently approved for use in the United States [10].

In an attempt to improve echocardiographic analysis, SQE has been performed in addition to QE. However, there is concern that PVL classification by SQE circumferential extent per VARC II criteria overestimates postTAVR PVL severity as compared to quantitative Doppler $[6,19,20]$ and CMR quantification [21]. Although technical limitations precluded routine quantitative Doppler in our series of symptomatic patients, we also found overestimation of PVL severity by SQE compared to CMR, with greater than $80 \%$ of patients classified as having greater than mild PVL by SQE compared to $48 \%$ by CMR. These findings are in concordance with those of Ribiero et al. [19], who found that $38 \%$ of their asymptomatic post-TAVR patients had greater than mild PVL by SQE compared to $24 \%$ by CMR. Our study also demonstrated inferior prognostic power of SQE compared to CMR.

Our study demonstrates that greater than mild postTAVR PVL by CMR is associated with a worse prognosis in a symptomatic population. This finding is supported by data from Merten et al. demonstrating that patients with no or mild post-TAVR PVL by CMR undergo beneficial LV structural remodeling, while those with greater than mild PVL do not [9]. A left ventricle that has chronically remodeled to facilitate pressure overload from aortic stenosis is expected to poorly tolerate moderate or severe regurgitation. Symptoms related to post-TAVR PVL might

Table 4 Follow-up Primary Composite Outcomes

\begin{tabular}{|c|c|c|c|c|}
\hline & $\begin{array}{l}\text { All } n=23 \\
(\%)\end{array}$ & $\begin{array}{l}\mathrm{RF} \leq 20 \% \\
\mathrm{n}=11(\%)\end{array}$ & $\begin{array}{l}\mathrm{RF}>20 \% \\
\mathrm{n}=12(\%)\end{array}$ & P value* \\
\hline Outcomes & $11(48)$ & $1(9)$ & $10(83)$ & 0.001 \\
\hline $\begin{array}{l}\text { Intractable heart failure } \\
\text { symptoms necessitating } \\
\text { repeat invasive therapy }\end{array}$ & $8(35)$ & $0(0)$ & $8(67)$ & \\
\hline $\begin{array}{l}\text { Heart failure } \\
\text { hospitalization }\end{array}$ & $4(17)$ & $0(0)$ & $4(33)$ & \\
\hline All-cause death & $5(22)$ & $1(9)$ & $4(33)$ & \\
\hline
\end{tabular}

*For Fisher's exact test.

$\mathrm{RF}=$ cardiovascular magnetic resonance derived regurgitant fraction.

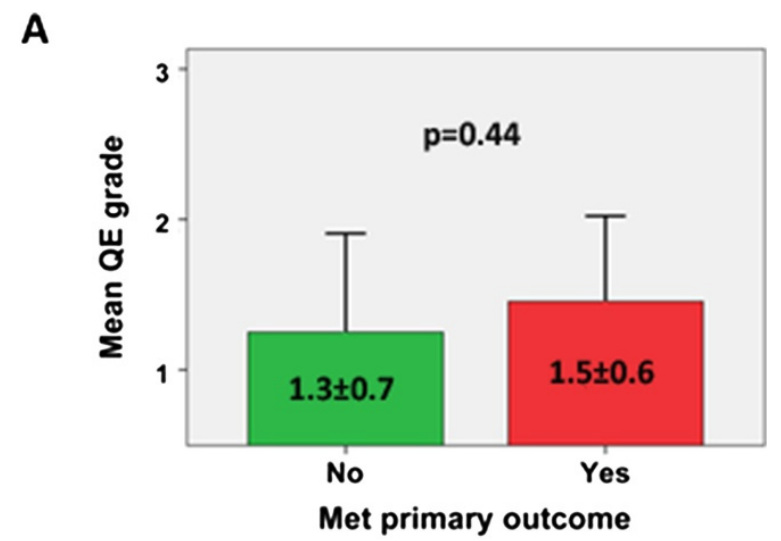

B

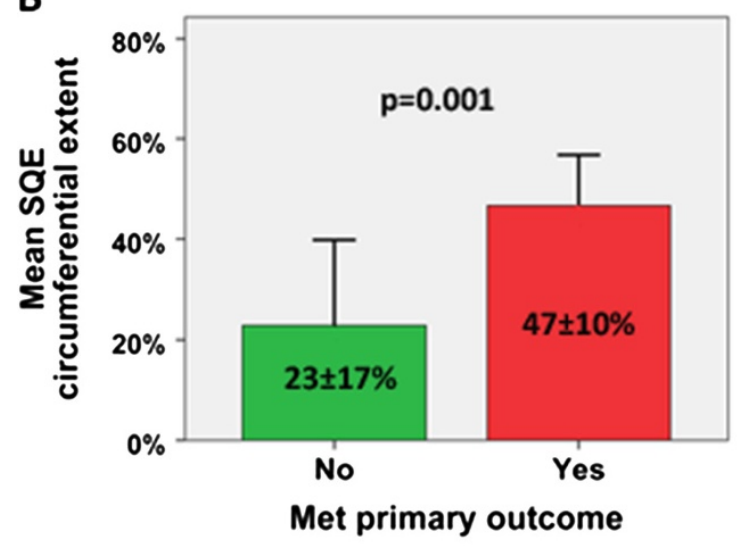

C

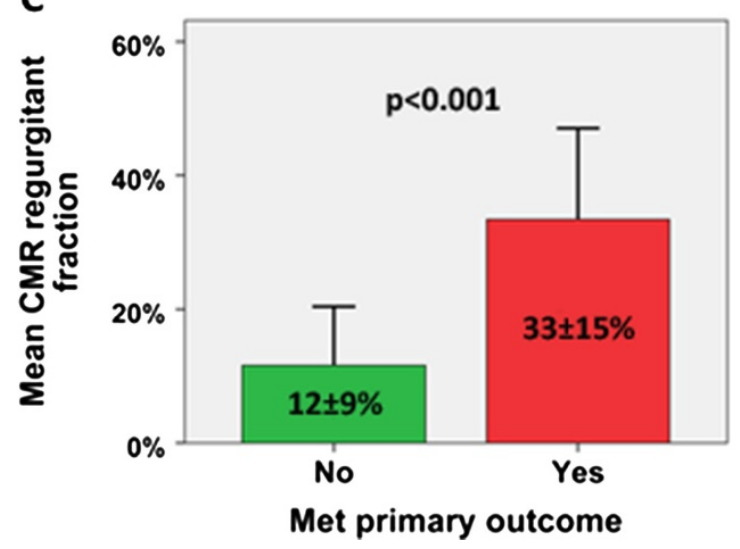

Figure 5 Comparison of paravalvular leak (PVL) severity in patients with different primary composite outcomes by imaging method. (A) Qualitative echocardiography (QE): 1 = mild, 2 = moderate, 3 = severe. (B) Semi-quantitative echocardiography (SQE) circumferential extent: mild $(<10 \%)$, moderate $(10-30 \%)$, and severe (>30\%). (C) Cardiovascular magnetic resonance (CMR) regurgitant fraction: mild $(\leq 20 \%)$, moderate $(21-39 \%)$, and severe $(\geq 40 \%)$. Primary composite outcome $=$ repeat invasive therapy, heart failure hospitalization, and all-cause death. P values are for independent samples t-test. 

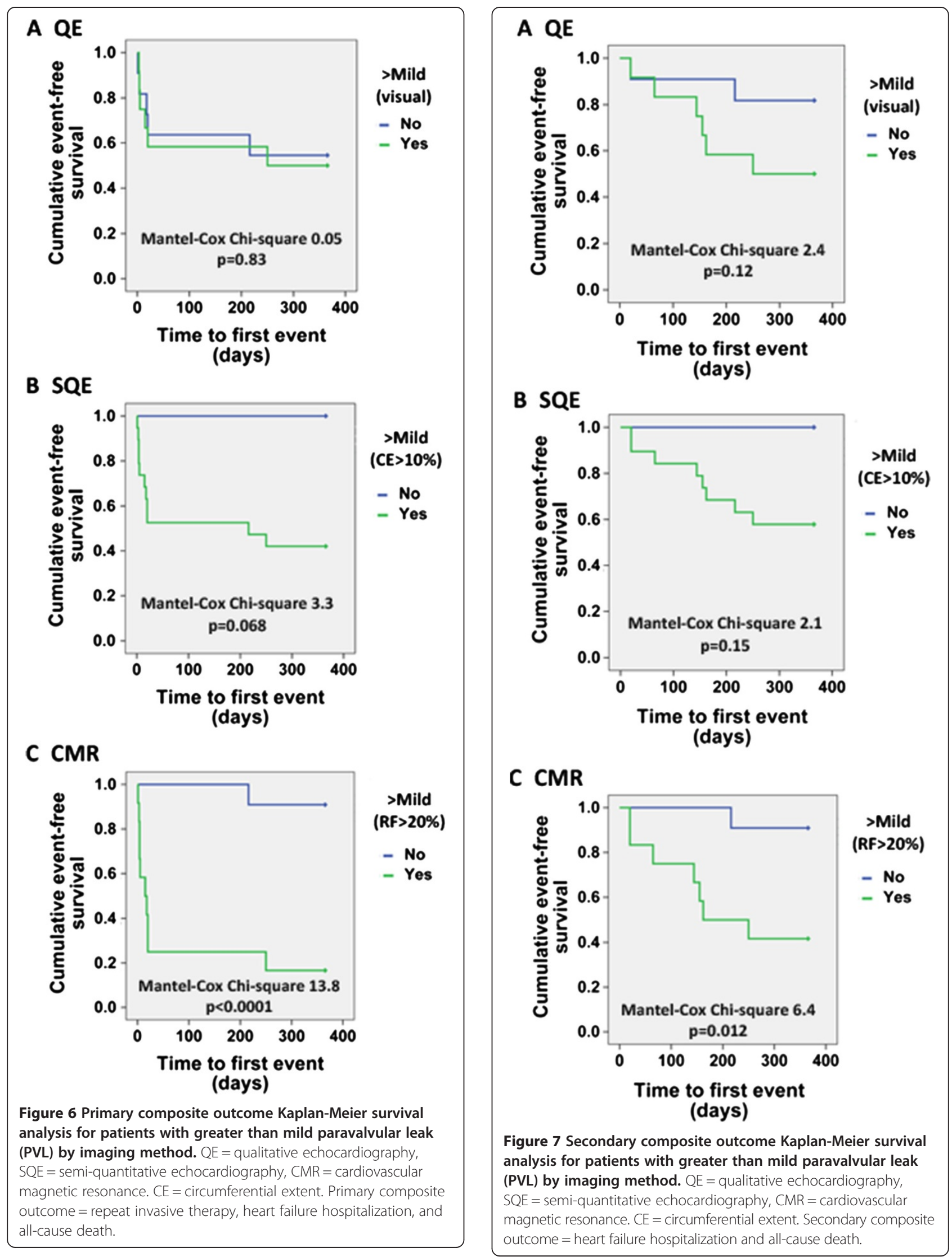
be difficult to differentiate from those related to systolic or diastolic heart failure and we believe CMR adds incremental value in this situation [22]. Our initial findings utilizing highly reproducible CMR quantification support further prospective validation of CMR for the evaluation and prognostication of post-TAVR PVL in a larger population of TAVR patients.

\section{Limitations}

The primary limitations of our study are the small number of patients from a single institution and retrospective collection of data. Small sample size may contribute to limited power to draw conclusive results, however this issue may be mitigated by the high event rate (48\%) for the primary composite outcome in this highly symptomatic cohort. The inclusion of symptomatic post-TAVR patients from our single institution also limits external validity, so generalization beyond our sample must be done with caution. Retrospective analysis may not account for unidentified confounders associated with poor outcomes post-TAVR, however, all patients were taking part in parallel prospective TAVR trials or registries in which meticulous follow-up and data collection were ensured.

Our primary composite outcome included repeat invasive therapy for refractory heart failure symptoms due to PVL, which could be driven by CMR findings and thus cause post-test bias. The secondary composite outcome, which did not include repeat invasive therapy, was significant for patients with $>20 \%$ RF by CMR. Many of our patients had difficult or suboptimal echocardiography. Semi-quantitative assessment using descending aortic flow reversal and quantitative assessment using right ventricular outflow tract or pulmonary artery Doppler were not routinely performed. This deficit likely reflects a modality specific shortcoming, as even experienced echocardiography centers report a substantial rate of technically difficult routine studies [23].

\section{Conclusions}

CMR stratifies post-TAVR PVL severity and should be considered early in the evaluation of symptomatic postTAVR patients. CMR is highly reproducible and commonly reclassifies PVL grade compared with qualitative and semi-quantitative TTE. Patients with greater than mild PVL by CMR (RF > 20\%) had a higher incidence of the primary composite outcome of all-cause death, heart failure hospitalization and intractable heart failure symptoms necessitating repeat invasive therapy.

\section{Competing interests}

Drs. Babaliaros, Thourani, and Lerakis are consultants for Edwards Lifesciences.

\section{Authors' contributions}

$\mathrm{GRH}$ contributed to the conception and design, acquisition of data, analysis and interpretation of data, and drafted the manuscript. VCB contributed to conception and design, analysis and interpretation of data, and made critical draft revisions. VHT contributed to interpretation of data and made critical draft revisions. SH contributed to acquisition of data and made critical draft revisions. CC contributed to interpretation of data and made critical draft revisions. NG performed the statistical analysis and made critical draft revisions. AES contributed to interpretation of data and made critical draft revisions. SDC contributed to interpretation of data and made critical draft revisions. JNO contributed to interpretation of data and made critical draft revisions. SL contributed to the conception and design, analysis and interpretation of data, and made critical draft revisions. All authors read and approved of the final manuscript.

\section{Authors' information}

GRH - Cardiology fellow (multimodality cardiovascular imaging). VCB Co-director, transcatheter valvular interventions and structural heart disease therapies (interventional cardiology). VHT - Co-director, transcatheter valvular interventions and structural heart disease therapies (cardiothoracic surgery). $\mathrm{SH}$ - Cardiology fellow (clinical investigator track). CC - General cardiologist. NG - Cardiology fellow (clinical investigator track). AES - Director, division of cardiothoracic radiology. SDC - Director, inpatient echocardiography lab. JNO - Director, magnetic resonance research. SL - Director of cardiovascular imaging for the emory structural and valve center, and Director of CMR and interventional echocardiography.

\section{Acknowledgements}

No funding sources.

\section{Author details}

${ }^{1}$ Department of Medicine, Division of Cardiology, Structural Heart and Valve Center, Emory University School of Medicine, Atlanta, Georgia. ${ }^{2}$ Department of Radiology and Imaging Science, Emory University School of Medicine, Atlanta, GA, Georgia. ${ }^{3}$ Department of Surgery, Division of Cardiothoracic Surgery, Structural Heart and Valve Center, Emory University School of Medicine, Atlanta, Georgia. ${ }^{4}$ Department of Biomedical Engineering, Georgia Institute of Technology/Emory University, Atlanta, Georgia. ${ }^{5}$ Emory University Hospital, 1365 Clifton Road NE, Suite AT503, Atlanta GA 30322, USA.

Received: 15 June 2014 Accepted: 12 November 2014

Published online: 05 December 2014

\section{References}

1. Kodali SK, Williams MR, Smith CR, Svensson LG, Webb JG, Makkar RR, Fontana GP, Dewey TM, Thourani VH, Pichard AD, Fischbein M, Szeto WY, Lim S, Greason KL, Teirstein PS, Malaisrie SC, Douglas PS, Hahn RT, Whisenant B, Zajarias A, Wang D, Akin JJ, Anderson WN, Leon MB, PARTNER Trial Investigators. Two-year outcomes after transcatheter or surgical aortic-valve replacement. N Engl J Med. 2012; 366:1686-95.

2. Makkar RR, Fontana GP, Jilaihawi H, Kapadia S, Pichard AD, Douglas PS, Thourani VH, Babaliaros VC, Webb JG, Herrmann HC, Bavaria JE, Kodali S, Brown DL, Bowers B, Dewey TM, Svensson LG, Tuzcu M, Moses JW, Williams MR, Siegel RJ, Akin JJ, Anderson WN, Pocock S, Smith CR, Leon MB, PARTNER Trial Investigators: . Transcatheter aortic-valve replacement for inoperable severe aortic stenosis. N Engl J Med. 2012; 366:1696-704.

3. Athappan G, Patvardhan E, Tuzcu EM, Svensson LG, Lemos PA, Fraccaro C, Tarantini G, Sinning JM, Nickenig G, Capodanno D, Tamburino C, Latib A, Colombo A, Kapadia SR. Incidence, predictors, and outcomes of aortic regurgitation after transcatheter aortic valve replacement: meta-analysis and systematic review of literature. J Am Coll Cardiol. 2013; 61:1585-95.

4. Lerakis S, Hayek SS, Douglas PS. Paravalvular aortic leak after transcatheter aortic valve replacement: current knowledge. Circulation. 2013; 127:397-407.

5. Zamorano JL, Badano LP, Bruce C, Chan KL, Gonçalves A, Hahn RT, Keane MG La Canna G, Monaghan MJ, Nihoyannopoulos P, Silvestry FE, Vanoverschelde $J$, Gillam LD. EAE/ASE recommendations for the use of echocardiography in new transcatheter interventions for valvular heart disease. J Am Soc Echocardiogr. 2011; 24:937-65.

6. Kappetein AP, Head SJ, Genereux P, Piazza N, van Mieghem NM, Blackstone EH, Brott TG, Cohen DJ, Cutlip DE, van Es GA, Hahn RT, Kirtane AJ, Krucoff MW, Kodali S, Mack MJ, Mehran R, Rodés-Cabau J, Vranckx P, Webb JG, Windecker S, 
Serruys PW, Leon MB. Updated standardized endpoint definitions for transcatheter aortic valve implantation: the Valve Academic Research Consortium-2 consensus document. J Am Coll Cardiol. 2012; 60:1438-54.

7. Hundley WG, Bluemke DA, Finn JP, Flamm SD, Fogel MA, Friedrich MG, Ho VB, Jerosch-Herold M, Kramer CM, Manning WJ, Patel M, Pohost GM, Stillman AE, White RD, Woodard PK. ACCF/ACR/AHA/NASCI/SCMR 2010 expert consensus document on cardiovascular magnetic resonance: a report of the American College of Cardiology Foundation Task Force on Expert Consensus Documents. Circulation. 2010; 121:2462-508.

8. Myerson SG. Heart valve disease: investigation by cardiovascular magnetic resonance. J Cardiovasc Magn Reson. 2012; 14:7.

9. Merten C, Beurich HW, Zachow D, Mostafa AE, Geist V, Toelg R, Richardt G, Abdel-Wahab M. Aortic regurgitation and left ventricular remodeling after transcatheter aortic valve implantation: a serial cardiac magnetic resonance imaging study. Circ Cardiovasc Interv. 2013; 6:476-83.

10. Sherif MA, Abdel-Wahab M, Beurich HW, Stöcker B, Zachow D, Geist V, Tölg R, Richardt G. Haemodynamic evaluation of aortic regurgitation after transcatheter aortic valve implantation using cardiovascular magnetic resonance. Eurolntervention. 2011; 7:57-63.

11. Abdel-Wahab M, Mehilli J, Frerker C, Neumann FJ, Kurz T, Tölg R, Zachow D, Guerra E, Massberg S, Schäfer U, El-Mawardy M, Richardt G, CHOICE investigators. Comparison of balloon-expandable vs self-expandable valves in patients undergoing transcatheter aortic valve replacement: the CHOICE randomized clinical trial. JAMA. 2014; 311:1503-14.

12. Chatzimavroudis GP, Walker PG, Oshinski JN, Franch RH, Pettigrew RI, Yoganathan AP. Slice location dependence of aortic regurgitation measurements with MR phase velocity mapping. Magn Reson Med. 1997; 37:545-51.

13. Cawley PJ, Hamilton-Craig C, Owens DS, Krieger EV, Strugnell WE, Mitsumori L, D'Jang CL, Schwaegler RG, Nguyen KQ, Nguyen B, Maki JH, Otto CM. Prospective comparison of valve regurgitation quantitation by cardiac magnetic resonance imaging and transthoracic echocardiography. Circ Cardiovasc Imaging. 2013; 6:48-57.

14. Dahiya A, Bolen M, Grimm RA, Rodriguez LL, Thomas JD, Marwick TH. Development of a consensus document to improve multireader concordance and accuracy of aortic regurgitation severity grading by echocardiography versus cardiac magnetic resonance imaging. Am J Cardiol. 2012; 110:709-14.

15. Gabriel RS, Renapurkar R, Bolen MA, Verhaert D, Leiber M, Flamm SD Griffin BP, Desai MY. Comparison of severity of aortic regurgitation by cardiovascular magnetic resonance versus transthoracic echocardiography. Am J Cardiol. 2011; 108:1014-20.

16. Gelfand EV, Hughes S, Hauser TH, Yeon SB, Goepfert L, Kissinger KV, Rofsky NM, Manning WJ. Severity of mitral and aortic regurgitation as assessed by cardiovascular magnetic resonance: optimizing correlation with Doppler echocardiography. J Cardiovasc Magn Reson. 2006; 8:503-7.

17. Myerson SG, D'Arcy J, Mohiaddin R, Greenwood JP, Karamitsos TD, Francis JM, Banning AP, Christiansen JP, Neubauer S. Aortic regurgitation quantification using cardiovascular magnetic resonance: association with clinical outcome. Circulation. 2012; 126:1452-60.

18. Orwat S, Diller G-P, Kaleschke G, Kerckhoff G, Kempny A, Radke RM, Buerke B, Burg M, Schulke C, Baumgartner $\mathrm{H}$. Aortic regurgitation severity after transcatheter aortic valve implantation is underestimated by echocardiography compared with MRI. Heart Published Online First. 2014; 24: doi:10.1136/heartjnl-2014-305665.

19. Ribeiro HB, Le Ven F, Larose E, Dahou A, Nombela-Franco L, Urena M, Allende R, Amat-Santos I, Ricapito MD, Thébault C, Clavel MA, Delarochelliére R, Doyle D, Dumont E, Dumesnil JG, Pibarot P, Rodés-Cabau J. Cardiac magnetic resonance versus transthoracic echocardiography for the assessment and quantification of aortic regurgitation in patients undergoing transcatheter aortic valve implantation. Heart Published Online First. 2014; 14: doi:10.1136/ heartjinl-2014-305615

20. Genereux P, Head SJ, Hahn R, Daneault B, Kodali S, Williams MR, van Mieghem NM, Alu MC, Serruys PW, Kappetein AP, Leon MB. Paravalvular leak after transcatheter aortic valve replacement: the new Achilles' heel? A comprehensive review of the literature. J Am Coll Cardiol. 2013; 61:1125-36.

21. Hayek S, Sawaya F, Oshinski J, Lerakis S. Multiparametric Assessment of Post-Transcatheter Aortic Valve Repacement Paravalvular Regurgitation Grading by Transthoracic Echocardiography and Cardiac Magnetic Resonance. J Clin Exp Cardiolog. 2014; 5:2.
22. Lerakis S, Hayek S, Arepalli CD, Thourani V, Babaliaros V. Cardiac magnetic resonance for paravalvular leaks in post-transcatheter aortic valve replacement. Circulation. 2014; 129:e430-1.

23. Senior R, Dwivedi G, Hayat S, Lim TK. Clinical benefits of contrast-enhanced echocardiography during rest and stress examinations. Eur J Echocardiogr. 2005; 6(Suppl 2):S6-13.

doi:10.1186/s12968-014-0093-x

Cite this article as: Hartlage et al:: The role of cardiovascular magnetic resonance in stratifying paravalvular leak severity after transcatheter aortic valve replacement: an observational outcome study. Journal of Cardiovascular Magnetic Resonance 2014 16:93.

\section{Submit your next manuscript to BioMed Central and take full advantage of:}

- Convenient online submission

- Thorough peer review

- No space constraints or color figure charges

- Immediate publication on acceptance

- Inclusion in PubMed, CAS, Scopus and Google Scholar

- Research which is freely available for redistribution

Submit your manuscript at www.biomedcentral.com/submit
C) Biomed Central 